\title{
COMMENTARY
}

\section{Is renal antiaging possible?}

\author{
Sadayoshi Ito \\ Hypertension Research (2010) 33, 1110-1111; doi:10.1038/hr.2010.165; published online 23 September 2010
}

$\mathrm{C}$ hronic kidney disease (CKD), as defined $\checkmark$ by reduced glomerular filtration rate (GFR) $\left(<60 \mathrm{ml} / \mathrm{min}\right.$ per $\left.1.73 \mathrm{~m}^{2}\right)$ and/or the presence of renal damages for more than 3 months, is a significant health problem. Both reduced GFR and albuminuria have been shown to be independently associated with heightened cardiovascular events (CVE) and renal events in various populations. ${ }^{1,2}$ The incidence and prevalence of $\mathrm{CKD}$ are increasing worldwide. In Japan, approximately 13 million patients have $\mathrm{CKD}$, and this number is estimated to increase further in the future. ${ }^{3}$ One of the major reasons for increasing incidence and high prevalence of CKD is aging. Imai et al. ${ }^{3}$ reported that the prevalence of CKD is more than $30 \%$ in the Japanese general population at the age of 70 years or higher. In addition to $\mathrm{CKD}$, older individuals commonly have hypertension, and hypertension is one of the modifiable risk factors of CKD and cardiovascular disease. Although various guidelines recommend that the target blood pressure (BP) goal for CKD is blow $130 / 80 \mathrm{~mm} \mathrm{Hg}$, there is no convincing evidence to support this goal for elderly CKD patients.

Many epidemiological studies have shown that baseline $\mathrm{CKD}$ is associated with high CVE rates. Practicing physicians usually monitor kidney function over a period of years during antihypertensive treatment. However, it is not entirely clear whether changes in renal function are related to future CVE rates.

Hayashi et al. ${ }^{4}$ reported a subanalysis of Japanese Trial to Assess Optimal Systolic Blood Pressure in Elderly Hypertensive patients (JATOS) that would gives us important information regarding relationships among baseline and changes in renal function,

Professor S Ito is at the Tohoku University, 1-1 Seiryocho, Sendai 980-8574, Japan.

E-mail: db554@mail.tains.tohoku.ac.jp
CVE rates and the level of BP control. First of all, they found an increase, rather than a decrease, in estimated GFR (eGFR) in both strict BP control (below $140 \mathrm{~mm} \mathrm{Hg}$ ) and mild BP control $(140-160 \mathrm{~mm} \mathrm{Hg})$ groups. This is surprising, as renal function declines with aging in general population and hypertension is known to accelerate this process. The increases do not seem to be because of acute intrarenal hemodynamic changes induced by calcium channel blocker, efonidipine, as eGFR increased very little in the first 6 months and then gradually increased until the end of 24-month follow-up period. Age (older or younger than 75 years), the presence of diabetes mellitus or baseline CKD did not affect the increases in eGFR, whereas those with baseline proteinuria and/or very poor blood control (achieved BP >160 $\mathrm{mm} \mathrm{Hg}$ ) did not show increases in eGFR. Of note, however, disappearance of proteinuria during treatment was closely associated with increases in eGFR. Because eGFR tended to decrease in total population with baseline proteinuria, it may be that in those with baseline proteinuria and no reduction during follow-up period, eGFR decreased substantially. This observation reinforces the importance of proteinuria and its management for renal protection. Both strict control of $\mathrm{BP}$ and reduction of albuminuria are the two wellestablished strategies for renal protection. In this study, eGFR was increased in those without baseline proteinuria or even in those with mild control of BP. Consistent with this study, few recent observational studies report that eGFR can increase over a period of years in hypertensive patients. ${ }^{5}$ It would be of particular interest and importance to identify the factors associated with (or the mechanism responsible for) the long-term changes in eGFR. It should be mentioned, however, that the renal end points, as assessed by doubling of serum creatinine or end-stage renal disease requiring dialysis therapy, were experienced only in 17 patients during 2-year follow-up of more than 4000 patients. In Japan, the average age of patients starting chronic dialysis is about 65 years. In JATOS study, elderly subjects at the age of $65-85$ years with baseline serum creatinine $<1.5 \mathrm{mg}$ per $100 \mathrm{ml}$ were enrolled. Thus, the population enrolled was at a low renal risk to start with, and they were so-called 'survivors' as far as renal function was concerned. Nevertheless, this study shows a possibility that in certain population, renal function can be improved over a period of years, a process against the natural biological course of aging.

It was observed that baseline CKD had no influences on CVE rates, whereas changes in eGFR and proteinuria had significant impacts on CVE. ${ }^{4}$ The change in proteinuria is a wellestablished prognostic factor for CVE. However, clinical significance of the change in renal function has only recently been reported, ${ }^{6}$ and JATOS study is consistent with these previous studies. It should be pointed out that the patients with serum creatinine of $1.5 \mathrm{mg}$ per $100 \mathrm{ml}$ or higher were excluded, so that most of the patients had stage $3 \mathrm{CKD}$. Along with a relatively short observation period (2 years), this inclusion bias may obscure the significance of baseline CKD as a cardiovascular risk. However, a recent prospective observational study (Gonryo CKD study) reported that baseline levels of kidney function were not independently related to CVE rates in CKD (stage 1-5) patients who had been well-treated with specialists $(n=2692$, average systolic and diastolic BP; $131 \pm 16$ and $77 \pm 11 \mathrm{~mm} \mathrm{Hg}$, respectively), and that the cause of renal disease (primary renal disease $v s$. hypertensive renal disease or diabetic nephropathy) had a great impact on CVE rates and total mortality. ${ }^{7}$ Thus, the issue of whether baseline GFR is an independent predictor of future CVE should be further evaluated under various conditions, particularly in the settings of clinical practice. 
Decrease in eGFR was associated with high CVE rates, particularly in CKD patients. However, it was unclear whether an increase in GFR resulted in a decreased CVE rate. In those with baseline CKD, CVE rates seemed to be numerically low in the case of substantial increase in eGFR $(>20 \%)$ as compared with the case of no change in eGFR $(-10$ to $+10 \%)$. Further investigation is needed to clarify whether improvement of GFR can reduce cardiovascular morbidity and mortality.

CVE rate did not differ in the presence or absence of baseline CKD, nor did it differ between strict BP control and mild BP control groups. However, poor control of BP (achieved BP >160 $\mathrm{mm} \mathrm{Hg}$ ) was associated with high CVE rates in both CKD and nonCKD patients. In addition, baseline proteinuria was associated with worse outcomes, especially in CKD patients, whereas diminution of proteinuria was associated with improved cardiovascular outcomes. Thus, CVE rate was highest in those with baseline proteinuria that persisted during the follow-up period. Although this study does not support intensive BP control $(<130 /$ $80 \mathrm{~mm} \mathrm{Hg}$ ) in elderly CKD patients, the results of this study cannot be extrapolated to more advanced proteinuric CKD patients, because the study included only a small number of proteinuric patients (15\%). However, the study has clearly shown that BP can be safely reduced to a level below $140 \mathrm{~mm} \mathrm{Hg}$.

Efonidipine was used as a baseline treatment in this study. It blocks L-type as well as T-type calcium channels, and has unique features as compared with pure L-type calcium channel blockers. Efonidipine has been shown to reduce proteinuria, inhibit aldosterone secretion, increase renal medullary blood flow ${ }^{8}$ and promotes natriuresis. Previous studies reported that in proteinuric patients already treated with an ACE inhibitor, further reduction of BP with addition of L-type calcium channel blocker did not confer any renal benefits. ${ }^{9}$ Interestingly, proteinuria disappeared in as many as $60 \%$ of patients with baseline proteinuria, and the rate of disappearance was not different between mild BP control group and strict BP control group, suggesting BP-independent mechanisms. Whether this antiproteinuric effect of treatment regimens is attributable to unique features of efonidipine or concomitant use of inhibitors of renin-angiotensin system remains to be determined.

In conclusion, the renal subanalysis of JATOS study has shown that antihypertensive therapy with efonidipine-based regimens improves kidney function independently of systemic BP. Furthermore, the incidence of CVE is greater in the subgroup with proteinuria or more marked decline in eGFR. Thus, the importance of monitoring both renal function and urinary protein excretion can never been overemphasized. Reduction of proteinuria still remains to be a main strategy for renal and cardiovascular protection in CKD patients even if they are old. Finally, it is of note that improvement of GFR can be possible against the natural biological course of aging. Clarification of the mechanism of the improvement and its clinical significance should await further investigation.
1 Hemmelgarn BR, Manns BJ, Lloyd A, James MT, Klarenbach S, Quinn RR, Wiebe N, Tonelli M. Relation between kidney function, proteinuria, and adverse outcomes. JAMA 2010; 303: 423-429.

2 Chronic Kidney Disease Prognosis Consortium Matsushita K, van der Velde M, Astor BC, Woodward M, Levey AS, de Jong PE, Coresh J, Gansevoort RT. Association of estimated glomerular filtration rate and albuminuria with all-cause and cardiovascular mortality in general population cohorts: a collaborative metaanalysis. Lancet 2010; 375: 2073-2081.

3 Imai E, Horio M, Watanabe T, Iseki K, Yamagata K, Hara S, Ura N, Kiyohara Y, Moriyama T, Ando Y, Fujimoto S, Konta T, Yokoyama H, Makino H, Hishida A, Matsuo S. Prevalence of chronic kidney disease in the Japanese general population. Clin Exp Nephrol 2009; 13: 621-630.

4 Hayashi K, Saruta T, Goto Y, Ishii M. Impact of renal function on cardiovascular events in elderly hypertensive patients treated with efonidipine. Hypertens Res 2010; 33: 1211-1220.

5 Ito S, Naritomi H, Ogihara T, Shimada K, Shimamoto K, Tanaka H, Yoshiike N. Increase in GFR during antihypertensive treatment is associated with reduced cardiovascular events in Japanese CKD patients: from J-HEALTH study. World Congress of Nephrology 2009. 22-26 May, Milan.

6 Matsushita K, Selvin E, Bash LD, Franceschini N, Astor $\mathrm{BC}$, Coresh J. Change in estimated GFR associates with coronary heart disease and mortality. J Am Soc Nephrol 2009; 20: 2617-2624.

7 Nakayama M, Sato T, Sato H, Yamaguchi Y, Obara K, Kurihara I, Sato K, Hotta O, Seino J, Miyata M, Takeuchi K, Nakayama K, Matsushima M, Otaka T, Kinoshita Y, Taguma $Y$, Ito S. Different clinical outcomes for cardiovascular events and mortality in chronic kidney disease according to underlying renal disease: the Gonryo study. Clin Exp Nephrol 2010; 14: 333-339.

8 Hu C, Mori T, Yoneki Y, Ohsaki Y, Nakamichi T, Ito S. Role of specific T-type calcium channel blocker R(-) efonidipine in regulation of medullary circulation. Hypertension 2008; 52: e34-131.

9 Ruggenenti P, Perna A, Loriga G, Ganeva M, Ene-lordache B, Turturro M, Lesti M, Perticucci E, Chakarski IN, Leonardis D, Garini G, Sessa A, Basile C, Alpa M, Scanziani R, Sorba G, Zoccali C, Remuzzi G. Blood-pressure control for renoprotection in patients with non-diabetic chronic renal disease (REIN-2): multicentre, randomised controlled trial. Lancet 2005; 365: 939-946. 